\title{
Adsorption and Desorption Behaviors of Cationic Liposome-DNA Complexes upon Lipofection in Inside and Outside Biomembrane Models Using a Dynamic Quasi-Elastic Laser Scattering Method
}

\author{
Yoshiko UChIYAma, ${ }^{* * *}$ Hiroharu YUI, ${ }^{*, * * * \dagger}$ and Tsuguo SAwada $* * * *$ \\ *Department of Advanced Materials Science, Graduate School of Frontier Sciences, The University of Tokyo, \\ Kibantou 603, 5-1-5 Kashiwanoha, Kashiwa, Chiba 277-8561, Japan \\ **Japan Society for the Promotion of Science (JSPS) \\ ***Core Research for Evolutional Science and Technology (CREST), Japan Science and Technology Agency \\ (JST), Nanoarchitectonics Research Center (NARC), National Institute of Advanced Industrial Science \\ and Technology (AIST), Tsukuba Central 4, 1-1-1 Higashi, Tsukuba, Ibaraki 305-8562, Japan
}

\begin{abstract}
The dynamic behaviors of cationic liposome-DNA complexes in inside and outside biomembrane models upon lipofection were investigated using the time-resolved quasi-elastic laser scattering (QELS) method. Inside and outside biomembrane models with similar phospholipid compositions to those in living cells were formed at a tetradecane/phosphate buffered saline (TD/PBS) interface. Cationic liposome-DNA complexes were injected into the buffer subphase, and their adsorption/desorption behaviors at the biomembrane models were monitored through changes in the interfacial tension. We found that the adsorption rate of the complexes increased 2.6 times more in the outside model than in the inside one. The adsorption rate of DNA alone did not show a remarkable difference from one side to the other; however, the adsorption rate of the cationic liposome alone showed a similar tendency to that of the liposome-DNA complex. These results indicated that the difference in lipid composition induced a different dynamic behavior of exogenous biomolecules and that the cationic liposomes played an important role in the faster incorporation of DNA into cells upon lipofection.
\end{abstract}

(Received August 2, 2004; Accepted September 13, 2004)

\section{Introduction}

Biomembranes play important roles in mass transfer, molecular recognition, and energy flow on membrane surfaces. Biomembranes are composed of many kinds of phospholipids and form bilayers by stacking the hydrophobic alkyl chain parts on the inside of the membrane. The major phospholipids in the cell organelle are phosphatidylethanolamine (PE), phosphatidylserine (PS), phosphatidylcholine (PC), sphingomyelin (SM), and phosphatidylinositol (PI). Interestingly, the composition of these phospholipids is asymmetric on the inside and outside of the cell membrane. The composition depends on the kind of cell and on the organelles. ${ }^{1,2}$ In general, the major parts of the PE and PS are in the inner half of the lipid bilayer, while PC and SM are mainly observed in the outer half. ${ }^{1,2}$ The different constitutions induce different structures and properties of biomembranes; such structures and properties are believed to be closely related to the recognition and selective transfer of exogenous biomolecules on the cell surfaces. However, the mechanisms governing the asymmetric constitution in the inside and outside monolayers are not clearly understood. The way in which they induce different dynamic behaviors of exogenous biomolecules into cells is not clear. Thus, it is intriguing and important to investigate the differences in the dynamic behaviors of

\footnotetext{
† To whom correspondence should be addressed.

E-mail: yui@molle.k.u-tokyo.ac.jp
}

exogenous biomolecules on the inner and outer surfaces of cells in order to understand how exogenous molecules are recognized and incorporated into the bilayer of a biomembrane.

Lipofection is one of the most powerful techniques of DNA transfection into living cells by the use of liposomes. Among various kinds of liposomes, cationic ones are efficient in gene transfer because they can strongly interact with DNAs and can spontaneously form cationic liposome-DNA complexes. They fuse the plasma membrane of cells and facilitate the delivery of functional DNA into the nucleus at high probability. ${ }^{3-11}$ Many studies have reported on the improvement of the transfection efficiency as a result of the development of synthetic cationic liposome. ${ }^{3-11}$ These studies were significant in the investigations of gene function ${ }^{12-18}$ and gene therapy ${ }^{19-30}$ in a broad range of diseases; the processes are safe, simple, and non-immunogenic.

A number of studies of DNA, cationic liposomes, and their complexes at solid/liquid, air/liquid, and liquid/liquid interfaces have been conducted to investigate the structures and properties of DNA-surfactant complexes at interfaces using UV-visible spectrophotometry, ${ }^{31-34}$ X-ray, ${ }^{35,36}$ ellipsometry, ${ }^{37,38}$ surface pressure,${ }^{39}$ the Du Nouy ring surface tensiometer, ${ }^{40}$ the spinning drop interfacial tensiometer, ${ }^{41}$ and the Wilhelmy method. ${ }^{42}$ These studies provided useful information on the static environment of the membrane. However, little is known about the dynamic behaviors of DNA carriers on membranes upon lipofection, which takes place on millisecond-to-second timescales. Furthermore, research on how the different constitutions inside and outside the biomembrane affect lipofection has not produced clear-cut answers. Thus, in situ 


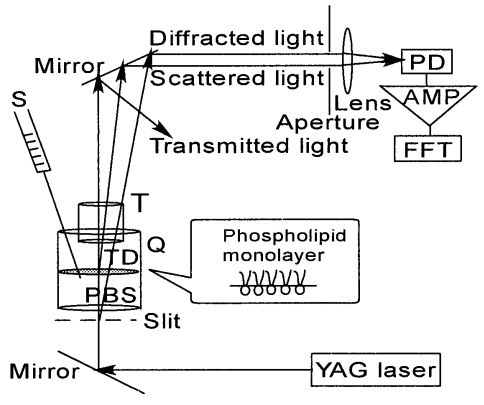

Fig. 1 Schematic diagram of the experimental setup for the QELS measurements: T, glass tube; $\mathrm{Q}$, quartz cell; S, microsyringe; PD, photodiode; AMP, preamplifier; FFT, FFT analyzer.

and time-resolved measurements of the dynamic behaviors of DNA carriers in a biomembrane model should provide important insights into the way in which a DNA carrier fuses with the bilayers of a biomembrane upon lipofection.

Here, we focused on the dynamic behaviors of external molecules in inside and outside biomembrane models upon lipofection. The differences in the dynamic behaviors of cationic liposome-DNA complexes were investigated. Such differences were induced by variances in the phospholipid constitutions between inside and outside biomembrane models. The inside and outside biomembrane models were formed individually with a tetradecane (TD)/phosphate-buffered saline (PBS) interface. The compositions were similar to those observed in the inside and outside of phospholipids in biological cells. A time-resolved quasi-elastic laser-scattering (QELS) method was used to monitor the adsorption and desorption of cationic liposome-DNA complexes in the models. The QELS method, which we have developed and applied to the study on the dynamics of mass transfer at liquid/liquid interfaces, ${ }^{43-57}$ allows non-perturbative in situ measurements of adsorption and desorption behaviors at liquid/liquid interfaces with a time resolution of $1 \mathrm{~s}$. These studies are expected to provide fundamental information on the adsorption and desorption behaviors of cationic liposome-DNA complexes on the inner and outer membrane surfaces in lipofection. In addition, they should clarify the phenomena of adsorption and desorption during the transfer across biomembranes, such as that in endocytosis and exocytosis, which are generally observed on cell surfaces.

\section{Experimental}

\section{Principle}

The principle of the QELS method has been reported elsewhere. ${ }^{58,59}$ In brief, an incident laser beam passing through the interface is scattered quasi-elastically with a Doppler shift by the capillary wave at the interface. The scattered beam is optically mixed with a local beam produced by a slit to generate an optical beat in the mixed light. The obtained beat frequency is the same as the Doppler shift, i.e., the capillary wave frequency.

The wave number of capillary wave $\kappa$ for the slit is expressed by the following equation, using the slit width $d=0.3 \mathrm{~mm}$ and the order $n$ of the diffraction spot. ${ }^{55,56,60-62}$

$$
\kappa=\frac{2 \pi(n+1 / 2)}{d}
$$

Table 1 Composition of phospholipids in the inside and outside biomembrane models

\begin{tabular}{lrrrrr}
\hline & PE & PS & PC & SM & PI \\
\hline Inside (\%) & 40 & 18 & 33 & 6 & 3 \\
Outside (\%) & 9 & 3 & 44 & 31 & 13 \\
\hline
\end{tabular}

The interfacial tension $\gamma$ is calculated from the capillary wave frequency $f$ and Eq. (1) using Lamb's equation, ${ }^{63}$

$$
f=\frac{1}{2 \pi}\left(\frac{\gamma}{\rho_{\mathrm{w}}+\rho_{\mathrm{o}}}\right)^{1 / 2} \kappa^{3 / 2},
$$

where $\rho_{\mathrm{w}}$ and $\rho_{\mathrm{o}}$ are the densities of the water phase and the oil phase, respectively, and $\gamma$ is the interfacial tension. The errors of the capillary wave frequency and the interfacial tension are estimated as $\pm 0.1 \mathrm{kHz}$ and $\pm 0.2 \mathrm{mN} / \mathrm{m}$, respectively.

\section{Apparatus}

A schematic diagram of the experimental setup is shown in Fig. 1. The light source is a $20 \mathrm{~mW}$ YAG laser (CrystaLaser, Model GCL-025S) with a wavelength of $532 \mathrm{~nm}$. A glass tube sets in the upper phase to extinguish the air-liquid interface. Both the quartz cell and the glass tube made of quartz glass have optically flat bottoms. After passing through the sample, one of the diffracted beams was selected by the aperture in front of the photodiode (Hamamatsu Photonics S1290) and mixed with the scattered light. In the present study, the fifth-order diffracted beam was selected for the measurement. Signals from the photodiode were Fourier-transformed and saved by a digital spectrum analyzer (Sony Tektronix Co., Model 3056). The wavelength of the observed capillary wave was $5.5 \times 10^{-3}$ $\mathrm{cm}$.

\section{Preparation of the biomembrane models}

The ratios of the inside and outside phospholipid constitutions in the present study are shown in Table 1. The ratios were determined by referring to representative values of various cell organelles. $^{2} \quad$ Chloroform (Wako)/hexane (Wako) mixed solvents (volume ratio; 1:1) were prepared; into these, phospholipids (PE, PS, PC, SM, PI) (Sigma) were dissolved at the concentrations shown in Table 1 . Further, $1.2 \mathrm{mg} / \mathrm{ml}$ cholesterol (Sigma) and $0.6 \mathrm{mg} / \mathrm{ml} \alpha$-tocopherol (Wako) were also dissolved into the inside and outside biomembrane model solutions. First, the mixed solvents were spread at an air/1 ml PBS (Wako) interface by microinjection in each quartz cell until the number density of the lipid molecules reached approximately $2.5 \times 10^{6} / 1 \mu \mathrm{m}^{2}$, which is similar to that in a lipid monolayer in cell membranes. ${ }^{1}$ After vaporization of the solvents, insoluble monolayers of different phospholipid constitutions remained at the air/PBS interface. Then, $1 \mathrm{ml}$ TD (Wako) was gently poured onto each monolayer model formed in each quartz cell. The TD phase (alkyl chain length, 14; the same number as that in phospholipids) in the biomembrane models plays the role of a quasi-alkyl chain layer of the counterpart membrane in lipid bilayers in cells. ${ }^{57}$ These monolayers that distinctively formed at TD/PBS were investigated as inside and outside biomembrane models.

\section{Sample preparation}

A $1.6-\mu \mathrm{g}$ volume of DNA (Invitrogen, plasmid pCMV, SPORT- $\beta$ gal) was dissolved in $100 \mu$ l PBS (pH 7.4). A $100-\mu l$ volume of $\mathrm{PBS}$ with $4 \mu \mathrm{l}$ liposome (Invitrogen, 
Table 2 Change of interfacial tension in the inside and outside biomembrane models $(\mathrm{mN} / \mathrm{m})$

\begin{tabular}{lcclcc}
\hline & \multicolumn{2}{c}{ Inside } & & \multicolumn{2}{c}{ Outside } \\
\cline { 2 - 3 } \cline { 5 - 6 } \cline { 5 - 6 } & Minimum & Equilibrium & & Minimum & Equilibrium \\
\hline None & - & 7.3 & - & - & 5.3 \\
Complex & $2.2(-5.1)$ & $2.9(-4.4)$ & & $1.7(-3.6)$ & $2.4(-2.9)$ \\
DNA & $3.8(-3.5)$ & $4.4(-2.9)$ & & $2.5(-2.8)$ & $3.3(-2.0)$ \\
Liposome & $4.1(-3.2)$ & $5.1(-2.2)$ & & $2.2(-3.1)$ & $2.9(-2.4)$ \\
\hline
\end{tabular}

Lipofectamine ${ }^{\mathrm{TM}}$ 2000) was also prepared. A DNA-liposome complex solution was made by mixing $100 \mu \mathrm{l}$ of the diluted DNA buffer solution and $100 \mu \mathrm{l}$ of the liposome buffer solution. To observe the dynamic behavior of DNA, liposome, and their complex in the biomembrane models, each component solution of each biomembrane model in the water phase was injected with a microsyringe. All chemicals were reagent grade and were used without further purification. All experiments were performed at room temperature $(298 \mathrm{~K})$.

\section{Results and Discussion}

To determine the initial values of the interfacial tension and to investigate the stability of the inside and outside biomembrane models, we measured the time courses of the capillary wave frequencies in those models formed at the TD/PBS interface (Fig. 2 (a)). No change in frequency was observed for at least 3 $\mathrm{h}$ in either case, indicating that both models were stable enough for the timescale of adsorption and/or desorption. The corresponding interfacial tensions for both models were calculated by Lamb's Eq. (2) and are plotted in Fig. 2 (b). The initial values of interfacial tension in the inside and outside biomembrane models were $7.3 \mathrm{mN} / \mathrm{m}$ and $5.3 \mathrm{mN} / \mathrm{m}$, respectively. The errors on these values were estimated within $0.1 \mathrm{mN} / \mathrm{m}$ from the standard deviation of 10 repeated measurements

To examine the dynamic behavior of cationic liposome-DNA complexes, we then measured the time courses of the interfacial tension in the inside and outside biomembrane models after the injection of cationic liposome-DNA complex solutions into the PBS phase. Changes in the surface tension due to the $\mathrm{ad} /$ desorptions of the surface-active molecules can be monitored by the time course of the capillary frequency induced by the change in interfacial tension with the relation shown in Eq. (2). In general, the adsorption of surface-active molecule induces a decrease in surface tension, and the desorption, in turn, induces an increase in surface tension. Thus, the first observed decrease in surface tension reflects the adsorption of the surface-active liposome-DNA complex, and the subsequent increase reflects the fact that some parts of the adsorbed phospholipid of liposomes and/or DNA were desorbed from the models. Unfortunately, the ratio of the desorbed phospholipid and DNA molecules cannot be determined using the QELS method alone. However, at least the difference in the total amount of adsorbed liposome-DNA complexes and their adsorption rate in the inside and outside biomembrane models can be discussed under the same injection conditions.

The time courses of interfacial tension after injection of the complexes are plotted in Fig. 3. When the cationic liposome-DNA complexes were injected in the outside biomembrane models, a gradual decrease of the capillary wave frequency was observed for about $12 \mathrm{~s}$. Then, it increased
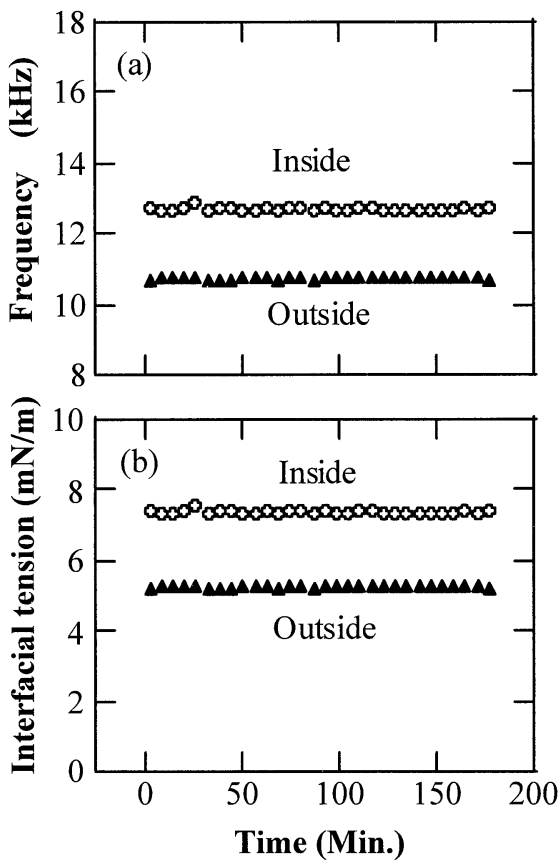

Fig. 2 Time courses of (a) capillary wave frequency and (b) interfacial tension of the inside and outside models.

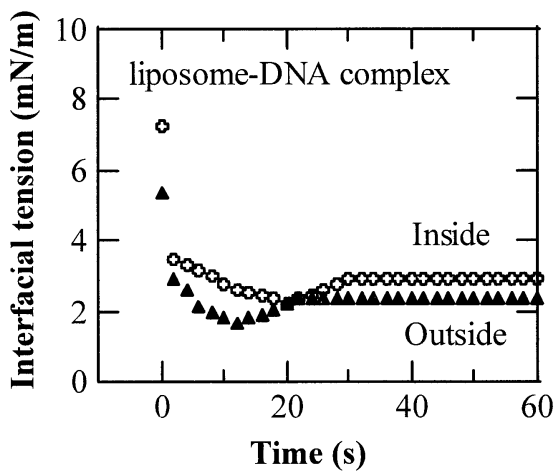

Fig. 3 Time courses of the interfacial tension of the inside and outside models after injection of a liposome-DNA complex solution.

gradually for $10 \mathrm{~s}$ and reached its equilibrium ( $>22 \mathrm{~s}$ after injection). The interfacial tension at the equilibrium was different from that measured before the injection by $2.9 \mathrm{mN} / \mathrm{m}$. This is because both liposome and DNA are surface-active and their adsorptions induce the decrease in the surface tension.

Next, we compared the dynamic behaviors of the cationic liposome-DNA complexes between the inside and outside biomembrane models. As shown in Fig. 3, we found that the dynamic behavior of the cationic liposome-DNA complex in the inside and outside biomembrane models remarkably differed, even though we had used the same injection condition. The difference in the dynamic behaviors of liposome-DNA complex is interesting because the different constitution of phospholipids in inside and outside biomembrane models induced the different adsorption and desorption behaviors of external biomolecules. The decrease in interfacial tension between the initial and minimum values and between the initial and equilibrium values and rates of decrease and increase in interfacial tension in both models are summarized in Tables 2 and 3. To avoid a negative influence on the rate estimation resulting from the flow induced 


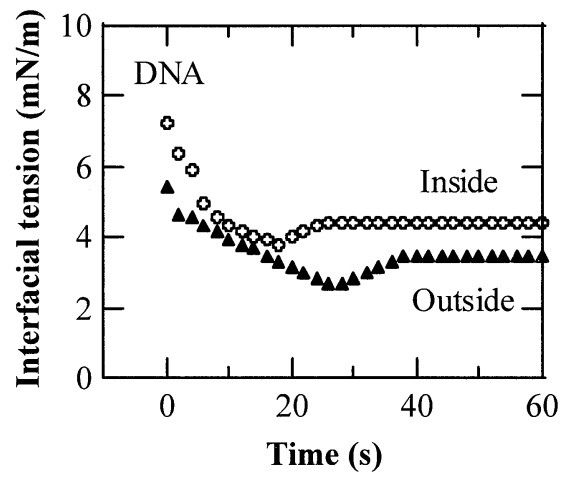

Fig. 4 Time courses of the interfacial tension of the inside and outside models after injection of a DNA solution.

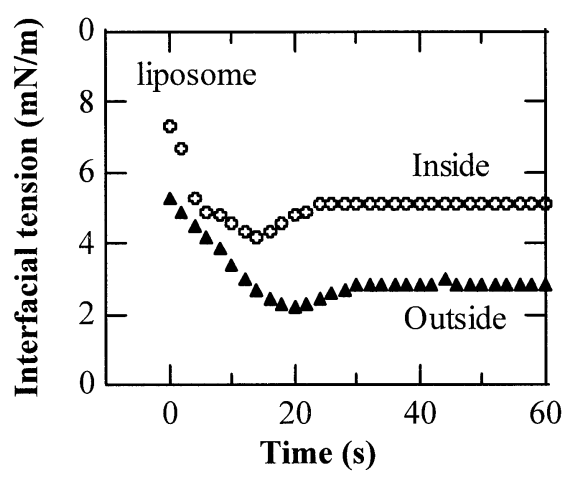

Fig. 5 Time courses of the interfacial tension of the inside and outside models after injection of a liposome solution.

by the injection, we applied data from $2 \mathrm{~s}$ after the injection to the time when interfacial tension reached its minimum, at the point when the decrease rate of interfacial tension was almost constant.

The changes in interfacial tension between the initial and minimum values, and between the initial and equilibrium values were 1.4 and 1.5 times larger in the inside model than those in the outside model, respectively. In general, the inside biomembrane consists of much more negatively charged phospholipids, such as PS. Thus, we can conclude that the reason for the larger number in the adsorption of the "cationic" liposome-DNA complex is due to the larger number of negatively charged phospholipids in the inside biomembrane. Interestingly, the adsorption rate of the cationic liposome-DNA complexes also showed a remarkable difference between the inside and outside models. In the outside model, the adsorption rate was 2.6 times larger than that observed in the inside model. In contrast, the rate of increasing tension of the complexes was almost the same for both models. Although the injection conditions were the same for both measurements, these results indicated that both the total amount of adsorption of the complex and the rate of the adsorption were different in the inside and outside biomembrane models. In particular, this remarkable difference in the adsorption rate is interesting because the incorporation process is considerably affected by the difference in the constitution of phospholipids in the biomembrane. Furthermore, although the total amount of negatively charged phospholipids is larger in the inside model, and the adsorption rate is larger in the outside model. This result indicates that the electrostatic interaction between the
Table 3 Change in interfacial tension under the adsorption and desorption of the DNA-liposome complex in the inside and outside biomembrane models

\begin{tabular}{lc}
\hline & Liposome-DNA complex \\
\hline & Inside $\quad$ Outside \\
$\Delta \gamma($ adsorption $)(\mathrm{mN} / \mathrm{m})$ & $5.1>3.6$ \\
$\Delta \gamma($ desorption $)(\mathrm{mN} / \mathrm{m})$ & $4.4>2.9$ \\
Adsorption rate $\left(\mathrm{mN} / \mathrm{m} \mathrm{s}^{-1}\right) \times 10$ & $0.8<2.1$ \\
Desorption rate $\left(\mathrm{mN} / \mathrm{m} \mathrm{s}^{-1}\right) \times 10$ & $0.7=0.7$ \\
\hline
\end{tabular}

Table 4 Comparison of the adsorption and desorption rates in the inside and outside biomembrane models

\begin{tabular}{lccc}
\hline & DNA & Liposome & $\begin{array}{c}\text { Liposome-DNA } \\
\text { complex }\end{array}$ \\
\hline & $\begin{array}{c}\text { Inside Outside } \\
0.8=0.8\end{array}$ & $\begin{array}{c}\text { Inside Outside } \\
1.0<1.8\end{array}$ & $\begin{array}{c}\text { Inside Outside } \\
0.8<2.1\end{array}$ \\
$\begin{array}{l}\text { Adsorption rate } \\
\left(\mathrm{mN} / \mathrm{m} \mathrm{s}^{-1}\right) \times 10\end{array}$ & $1.0>0.6$ & $0.7=0.7$ \\
$\begin{array}{l}\text { Desorption rate } \\
\left(\mathrm{mN} / \mathrm{m} \mathrm{s}^{-1}\right) \times 10\end{array}$ & $0.8=0.9$ & & \\
\hline
\end{tabular}

positively charged cationic liposome and the negatively charged phospholipid cannot explain such a difference in the dynamic behaviors.

To examine what induces such a remarkable difference in the rate of cationic liposome-DNA complexes in the inside and outside models, we separately measured the dynamic behaviors of DNA alone and of cationic liposome alone. The interfacial tensions after the injection of DNA and cationic liposome solutions in the inside and outside biomembrane models are plotted as a function of time in Figs. 4 and 5, respectively. A different dynamic behavior of DNA and cationic liposome was likewise generated in the inside and outside biomembrane models. The decreased interfacial tension between the initial and minimum values and that between the initial and stationary state values of DNA and cationic liposomes are summarized in Table 2. The adsorption and desorption rates of DNA alone, liposomes alone, and their complexes are summarized in Table 4. To compare the adsorption rates of the complexes, DNA, and cationic liposomes, the applied data are shown in Figs. 6 (a), (b), and (c), respectively. No remarkable difference was observed in the adsorption rate of DNA alone between the inside and outside biomembrane models, as shown in Fig. 6 (b). This showed that DNA had little influence on the different adsorption rate of the cationic liposome-DNA complexes in the inside and outside biomembrane models. This was considered to be due to the negatively charged DNA being effectively bound and surrounded by the cationic liposomes. In contrast, the adsorption rate of cationic liposomes alone in the outside biomembrane model was 1.8 times larger than that observed in the inside model, indicating that the cationic liposomes were faster at incorporating DNA into cells in phospholipid membranes with an outside constitution than in those with an inside constitution. This is similar to the dynamic behavior of the cationic liposome-DNA complex, as shown in Fig. 6 (a). These results indicate that not the DNA but the surrounding cationic liposomes of the complexes are closely related to the remarkable differences in the adsorption rates of the cationic liposome-DNA complexes between in the inside and outside biomembrane models. However, as mentioned, the electrostatic interaction between the cationic liposome and negatively 

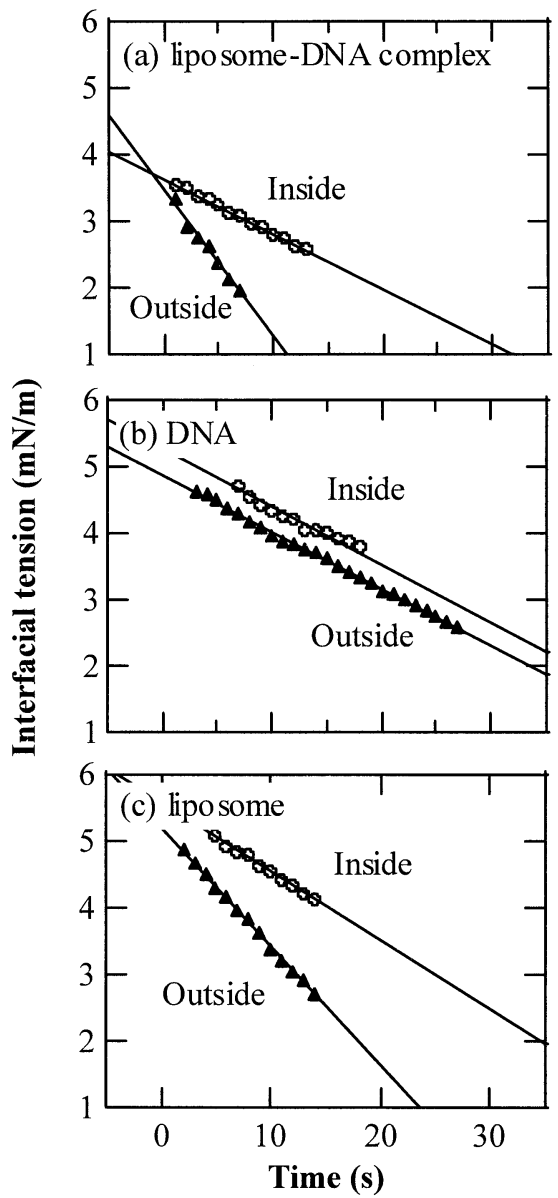

Fig. 6 Estimation of the adsorption rate of (a) liposome-DNA complex, (b) DNA, and (c) liposome in both the inside and the outside models. Solid lines in each panel indicate a fitted line to obtain the adsorption rate of the inside and outside models, respectively.

charged PS cannot explain such a tendency. To explain these features, we must take into account the role of the counter-ions adjacent to the charged phospholipid and the stability of the complex between the cationic liposomes and the negatively charged DNA molecules in the inside and outside models. Although the reason for the acceleration of the adsorption rate of the liposome-DNA complexes in the outside biomembrane models is not yet clear, it is interesting that such a different dynamic behavior was actually induced on the second timescale due to the asymmetric constitution of the biomembrane. Such real-time monitoring of the dynamic behaviors of exogenous molecules in biomembranes will contribute to clarify the biological role of the asymmetric constitutions of the inside and outside biomembranes.

\section{Summary}

We measured the adsorption and desorption behaviors of cationic liposome-DNA complexes in inside and outside biomembrane models using the dynamic QELS method. The different dynamic behaviors of cationic liposome-DNA complexes in the inside and outside models were successfully monitored. It was found that the adsorption rate of the cationic liposome-DNA complexes in the biomembrane model with an outside constitution was remarkably increased. From the reference experiments, the mutual interaction between cationic liposome and biomembrane was revealed to play an important role in accelerating the adsorption of the complexes. We expect that the QELS method will not only give valuable information on the design of cationic liposomes for efficient DNA incorporation, but also provide a useful tool to understand mass transfer phenomena across biomembranes.

\section{References and Notes}

1. B. Alberts, D. Bray, J. Lewis, M. Raff, K. Roberts, and J. D. Watson, "Molecular biology of the cell", 3rd ed., 1994, Chap. 10, Garland Publishing, USA, 10, 478.

2. Y. Nozawa, "Seitaimakunimanabu", 7th ed., 1997, Kyoritsu Publishing, 21.

3. P. L. Felgner, T. R. Gadek, M. Holm, R. Roman, H. W. Chan, M. Wenz, J. P. Northrop, G. M. Ringold, and M. Danielsen, Proc. Natl. Acad. Sci. USA, 1987, 84, 7413.

4. P. L. Felgner and G. M. Ringold, Nature, 1989, 337, 387.

5. J. Gaucheron, C. Santaella, and P. Vierling, Bioconjug. Chem., 2001, 12, 114.

6. O. Boussif, J. Gaucheron, C. Boulanger, C. Santaella, H. V. J. Kolbe, and P. Vierling, J. Gene Med., 2001, 3, 109.

7. J. H. Felgner, R. Kumar, C. N. Sridhar, C. J. Wheeler, Y. J. Tsai, R. Border, P. Ramsey, M. Martin, and P. L. Felgner, J. Biol. Chem., 1994, 269, 2550.

8. J.-P. Behr, B. Demeneix, J. P. Loeffler, and J. Perez-Mutul, Proc. Natl. Acad. Sci. USA, 1989, 86, 6982.

9. L. Roy, S. Cates, K. Schifferli, J.-P. Pichet, V. Ciccarone, S. Bennett, and P. Hawley-Nelson, Focus, 1999, 21, 62.

10. K. Fabio, J. Gaucheron, C. D. Giorgio, and P. Vierling, Bioconjug. Chem., 2003, 14, 358.

11. J. A. Heyes, D. Niculescu-Duvaz, R. G. Cooper, and C. J. Springer, J. Med. Chem., 2002, 45, 99.

12. S. Ohnuma, F. Mann, S. Boy, M. Perron, and W. A. Harris, Methods, 2002, 28, 411.

13. Y. Watanabe, H. Nomoto, R. Takezawa, N. Miyoshi, and T. Akaike, J. Biochem., 1994, 116, 1220.

14. T. Inazawa, T. Tanabe, H. Yamada, T. Nakaoka, Y. Hashimoto, T. Yamasaki, H. Kotaki, K. Tani, S. Asano, and N. Yamashita, Mol. Ther., 2001, 4, 267.

15. Y. Wakamatsu, Y. Watanabe, H. Nakamura, and $H$. Kondoh, Development, 1997, 124, 1953.

16. X. Liang, A. Teng, D. M. Braun, J. Felgner, Y. Wang, S. I. Baker, S. Chen, O. Zelphati, and P. L. Felgner, J. Biol. Chem., 2002, 277, 3593.

17. H. Nakamura, Y. Watanabe, and J-I. Funahashi, Develop. Growth Differ., 2000, 42, 199.

18. L. Vallier, P. J. Rugg-Gunn, I. A. Bouhon, F. K. Andersson, A. J. Sadler, and R. A. Pedersen, Stem Cells., 2004, 22, 2.

19. S. Gaurang, M. D. Daftary, S. Hugh, and M. D. Taylor, Fertil. Steril., 2003, 80, 475.

20. R. M. Bright-Thomas, A. Agrawal, and R. Hargest, Brit. J. Surg., 2002, 89, 1563.

21. L. Bildirici, P. Smith, C. Tzavelas, E. Horefti, and D. Rickwood, Nature, 2000, 405, 298.

22. M. Hashida, M. Nishikawa, F. Yamashita, and Y. Takakura, Adv. Drug Deliv. Rev., 2001, 52, 187.

23. X. B. Zhao and R. J. Lee, Adv. Drug Deliv. Rev., 2004, 56, 1193.

24. C. R. Dass and M. A. Burton, J. Pharm. Pharmacol., 1999, 51,755 . 
25. E. Coveney, B. Clary, M. Iacobucci, R. Philip, and K. Lyerly, Surgery, 1996, 120, 265.

26. K. Son and L. Huang, Proc. Natl. Acad. Sci. USA, 1994, 91, 12669.

27. M. Yano, Y. Nakashima, Y. Kobayashi, K. Mizuno, A. Konishi, H. Sasaki, I. Fukai, R. K. Scheule, and Y. Fujii, Cancer. Gene Ther., 2004, 135, 193.

28. P. L. Felgner and G. Rhodes, Nature, 1991, 349, 351.

29. T. Ota, M. Maeda, and M. Tatsuka, Anticancer Res., 2002, 22,4049 .

30. S. Sturlan, A. Schneeberger, M. Fang, B. G. Beinhauer, P. Luhrs, R. Kutil, B. Wessner, L. Huang, A. O. Aasen, and M. A. Rogy, Anticancer Res., 2003, 23, 4843.

31. F. Ganachaud, A. Elaissari, and C. Pichot, Langmuir, 1997, 13,7021 .

32. A. S. Andreeva, M. O. Gallyamov, O. A. Pyshkina, V. G. Sergreev, and I. V. Yaminskii, Russ. J. Phys. Chem., 1997, 73(11), 1858.

33. M. Sastry, A. Kumar, M. Pattarkine, V. Ramakrishnan, and K. N. Ganesh, Chem. Commun., 2001, 16, 1434.

34. S. A. Gani, D. C. Mukherjee, and D. K. Chattoraj, Langmuir, 1999, 15, 7130.

35. K. Kago, H. Matsuoka, R. Yoshitome, H. Yamaoka, K. Ijiro, and M. Shimomura, Langmuir, 1999, 15, 5193.

36. I. Koltover, T. Salditt, J. O. Radler, and C. R. Safinya, Science, 1998, 281, 78.

37. K. Eskilsson, C. Leal, B. Lindman, M. Miguel, and T. Nylander, Langmuir, 2001, 17, 1666.

38. M. Cardenas, A. Bream, T. Nylander, and B. Lindman, Langmuir, 2003, 19, 7712.

39. X. Chen, J. Wang, N. Shen, Y. Luo, L. Li, M. Liu, and R. K. Thomas, Langmuir, 2002, 18, 6222.

40. W. J. Xia and H. Onyuksel, Pharm. Res., 2000, 17(5), 612.

41. T. F. Svitova, Y. P. Smirnova, S. A. Pisarev, and N. A. Berezina, Colloid. Surface A, 1995, 98, 107.

42. P. Boncuk, M. Kaser, Y. Yu, and H. W. Taeusch, Lipids, 1997, 32(3), 247.

43. S. Takahashi, A. Harata, T. Kitamori, and T. Sawada, Anal. Sci., 1991, 7(Supplement), 645.

44. S. Takahashi, A. Harata, T. Kitamori, and T. Sawada,
Bunseki Kagaku, 1991, 40, 761.

45. S. Takahashi, A. Harata, T. Kitamori, and T. Sawada, Anal. Sci., 1994, 10, 305.

46. Z. Zhang, I. Tsuyumoto, S. Takahashi, T. Kitamori, and T. Sawada, J. Phys. Chem. A, 1997, 101, 4163.

47. Z. Zhang, I. Tsuyumoto, S. Takahashi, T. Kitamori, and T. Sawada, J. Phys. Chem. B, 1998, 102, 10284.

48. I. Tsuyumoto, N. Noguchi, T. Kitamori, and T. Sawada, $J$. Phys. Chem. B, 1998, 102, 2684.

49. S. Takahashi, I. Tsuyumoto, T. Kitamori, and T. Sawada, Electrochim. Acta, 1998, 44, 165.

50. Y. Uchiyama, I. Tsuyumoto, T. Kitamori, and T. Sawada, J. Phys. Chem. B, 1999, 103, 4663.

51. Y. Uchiyama, M. Fujinami, T. Sawada, and I. Tsuyumoto, J. Phys. Chem. B, 2000, 104, 4699.

52. Y. Uchiyama, T. Kitamori, T. Sawada, and I. Tsuyumoto, Langmuir, 2000, 16, 6597.

53. T. Takahashi, H. Yui, and T. Sawada, J. Phys. Chem. B, 2002, 106, 2314.

54. H. Yui, Y. Ikezoe, T. Takahashi, and T. Sawada, J. Phys. Chem. B, 2003, 107, 8433 .

55. Y. Ikezoe, S. Ishizaki, T. Takahashi, H. Yui, M. Fujinami, and T. Sawada, J. Colloid Interface Sci., 2004, in press.

56. Y. Ikezoe, S. Ishizaki, H. Yui, M. Fujinami, and T. Sawada, Anal. Sci., 2004, 20, 435.

57. T. Morisaku, H. Yui, M. Iwazumi, Y. Ikezoe, M. Fujinami, and T. Sawada, Anal. Chem., 2004, 76, 2314.

58. H. Lofgren, R. D. Neuman, E. L. Scriven, and T. H. Davis, J. Colloid Interface Sci., 1984, 98, 175.

59. S. Hard and R. D. Neuman, J. Colloid Interface Sci., 1987, 115,73 .

60. S. Hard, Y. Hamnerius, and O. Nilsson, J. Appl. Phys., 1976, 47, 2433.

61. R. Kronig, "Textbook of Physics", 2nd ed., 1959, Pergamon Press, London, 428.

62. A. Trojanek, P. Krtil, and Z. Samec, Electrochem. Commun., 2001, 3, 613.

63. H. Lamb, "Hydrodynamics", 6th ed., 1932, Cambridge University Press, London. 